\title{
Ligating the left atrial appendage in atrial fibrillation: What additional evidence do surgeons need?
}

\author{
Dawn S. Hui, MD, and Richard Lee, MD, MBA
}

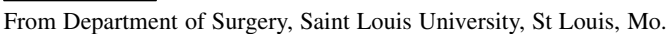 \\ Received for publication Sept 10, 2018; accepted for publication Sept 11, 2018; available ahead of print Nov 7, \\ 2018. \\ Address for reprints: Richard Lee, MD, MBA, Saint Louis University, 48 Picardy Ln, St Louis, MO 63124 \\ (E-mail: rlee@slu.edu). \\ J Thorac Cardiovasc Surg 2019;157:1004-6 \\ 0022-5223/ $\$ 36.00$ \\ Copyright $(c) 2018$ by The American Association for Thoracic Surgery \\ https://doi.org/10.1016/j.jtcvs.2018.09.041
}

In patients with atrial fibrillation (AF), there are 2 surgical mechanisms that have the potential to reduce stroke and stroke-related mortality: successful ablation with restoration of normal sinus rhythm and removal of the substrate for thrombi by surgical left atrial appendage occlusion (SLAAO). In patients with AF undergoing cardiac surgery for other reasons, surgeons have a unique opportunity to perform both.

The 2017 Society of Thoracic Surgeons (STS) guidelines currently recognize S-LAAO for concomitant cardiac operations as a class IIA recommendation, ${ }^{1}$ citing a 2015 metaanalysis of 3653 patients from 7 studies (including 2 randomized trials) in which S-LAAO was associated with significantly reduced stroke incidences at 30 days (odds ratio, 0.46) and latest follow-up (odds ratio, 0.48). ${ }^{2}$ In a subsequent study of patients with AF undergoing valvular surgery from the Nationwide Inpatient Sample Database, 652 case-controlled pairs matched for $\mathrm{CHA}_{2} \mathrm{DS}_{2}$-VASC score were compared. S-LAAO was significantly associated with decreased in-hospital cerebrovascular events, inhospital mortality, and length of stay. ${ }^{3}$

The recent study by Friedman and colleagues ${ }^{4}$ published in JAMA adds considerably to the growing evidence on S-LAAO. By linking data from the STS Adult Cardiac Database with Medicare claims data and the Medicare Denominator File, the authors were able to study more than 10,000 patients aged 65 years and older with AF undergoing cardiac surgery. S-LAAO was associated with significantly lower rates of rehospitalization for thromboembolism, all-cause mortality, and a composite end point of thromboembolism, hemorrhagic stroke, or all-cause mortality at a mean follow-up of 2.6 years. This extends the outcome period beyond previous studies, providing meaningful midterm outcomes for a highly relevant population

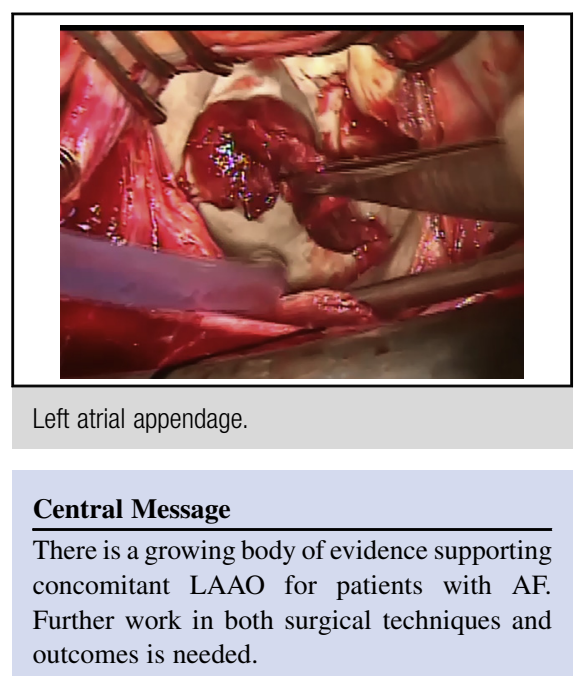

See Article page 999.

(ie, elderly and at high risk for stroke with a median $\mathrm{CHA}_{2}-\mathrm{DS}_{2}$-VASc score of 4 ).

As with any observational study, valid criticisms exist. Is what the study lacks in design (ie, nonrandomization) accounted for by statistical analysis? The authors put considerable effort toward adjusting for treatment bias, using inverse probability-weighted models and additionally examined for residual confounding by using falsification end points. Analyses were also conducted to exclude confounding by overall quality of care variation and concomitant surgical ablation. Beyond this, there are 2 important variables for which long-term data are lacking: the efficacy of rhythm management-whether by surgical or medical means-and long-term anticoagulation.

We propose that the weaknesses identified here should not dissuade surgeons from occluding the LAA when there is an opportunity to do so. The study by Friedman and colleagues ${ }^{4}$ shows that the adoption rate of S-LAAO is still low-37\%-among patients with known AF at the time of operation. Their findings should in fact prompt greater consideration of S-LAAO at concomitant operations. That the benefit was found among patients discharged without anticoagulation is important. The reality is that for many patients, especially elderly patients, oral anticoagulation may not be a viable long-term treatment option. First, it is known that in major trials, the rate of oral anticoagulation discontinuation was $21 \%$ to $34 \%$. $^{5}$ Real-world rates would be expected to be at least equivalent, if not higher. In fact, a study of commercially insured patients found that whereas novel 


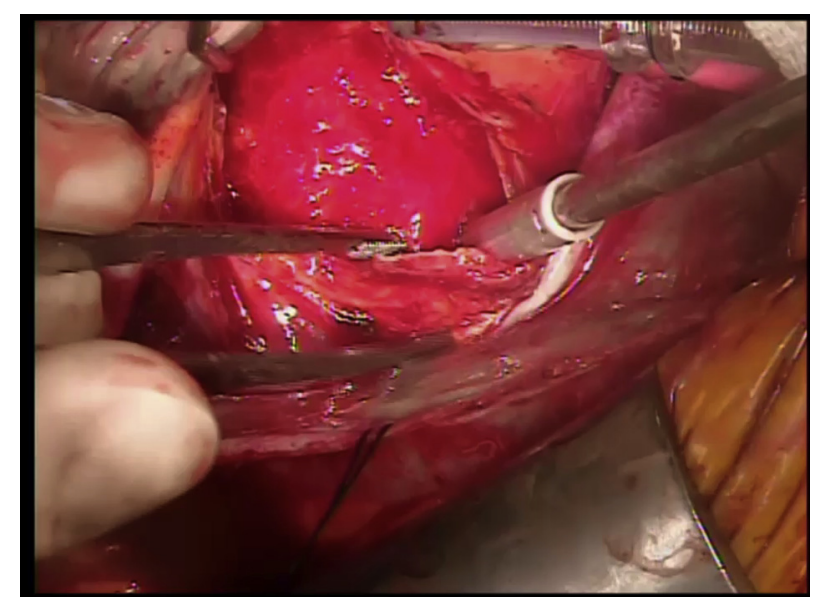

VIDEO 1. Surgical removal of a gigantic left atrial thrombus originating from the left atrial appendage. Video available at: https://www.jtcvs.org/ article/S0022-5223(18)32545-5/fulltext.

oral anticoagulants had a modestly improved adherence rate compared with warfarin, the rate for either class of medication was below $50 \%$ at a median of 1.1 years. ${ }^{6}$ Any bleeding complication is more likely than not to result in permanent discontinuation. No crystal ball offers a view of which patients will become nonadherent, whether by choice or by medical necessity. Although an implanted Watchman device (Boston Scientific, Marlborough, Mass) may be an option for these patients, it requires another procedure and short-term anticoagulation. Likely, a sentinel event will be the mechanism by which these patients are identified. Why not prevent complications at the time of surgery?

\section{CRITIQUES OF SURGICAL TECHNIQUE}

Critics are quick to point out inadequacy of surgical technique and make unfounded claims that incomplete ligation increases stroke. Admittedly, they are correct regarding a need to improve technique. In the surgical management of $\mathrm{AF}$ there has been steady progress in energy sources and efficacy of tools used for surgical ablation, as well as a growing body of literature on choice of lesion set. In contrast, less attention has been paid to S-LAAO. Scrutiny of surgical results is sorely needed. There are only a handful of studies looking specifically at the success of S-LAAO, ${ }^{7-11}$ and these have small cohort sizes (range, 28-130 patients). The rate of success has been as low as $0 \%$ for stapled exclusion ${ }^{10}$ and as high as $90 \%$ with internal ligation. ${ }^{8}$ A randomized trial comparing 3 standard techniques (eg, stapled excision, surgical excision, and internal suture ligation) with a $75 \%$ follow-up transesophageal echocardiogram rate showed an overall failure rate $>50 \%$ with no significant difference by technique. ${ }^{11}$ This study, ${ }^{11}$ as well as that conducted by Healey and colleagues, ${ }^{9}$ found that the mode of failure differed according to method: residual flow occurred with internal ligation due to suture dehiscence, and residual stumps occurred with external methods. Healey and colleagues ${ }^{9}$ found that surgeon experience correlated with success, although it should be noted that staplers were used with increasing frequency during the period of study. So what?

Strokes in patients with AF are associated with significantly larger infarct size, more severe hemorrhagic transformation, and higher mortality compared with strokes in patients without AF. ${ }^{12}$ This is likely due to the large thrombus burden (Video 1). This may be a mechanism for reduced mortality in strokes, even after failed ligation. In among the earliest articles identifying high rates of incomplete LAA ligation, Kanderian and colleagues ${ }^{10}$ found that success of LAAO was not associated with significantly decreased transient ischemic attack or stroke rate. However the sample size was small and, similar to Friedman and colleagues, ${ }^{4}$ there was no adjustment for late AF or oral anticoagulant use. Two other areas that may cloud comparisons include distinction between valvular and nonvalvular AF and the influence of left atrium size. In a series of 773 patients using a variety of LAA ligation techniques, there were 25 late neurologic events; on multivariable analysis, there was no difference by technique. ${ }^{13}$ More importantly, only 1 stroke led to a mortality. Usually strokes from AF are 3 times more fatal than from other causes. ${ }^{12}$ One explanation for this finding is a reduction of thrombus burden by eliminating the LAA, even if incomplete.

Eventually, low rates of successful S-LAAO do not have to be inevitable. Knowing the modes of S-LAAO failure provides a starting point for exploring techniques to improve outcomes. In fact, attention to technique of the internal ligation method has been described and discussed, ${ }^{8,14}$ but widespread adoption of such modifications is unknown at this time. An individualized approach accounting for concomitant procedures, exposure, anatomy, and tissue integrity is more important than whether any one particular method has evidence to support it as a superior method.

Inclusion of surgical technique will inform future research, including not only so-called success by echocardiographic findings, but also whether that success translates to improved outcomes of thromboembolism rates and mortality. The STS Adult Cardiac Database would be a logical place to begin collecting such data. In the treatment of AF, we have seen success in improving the rates of concomitant ablation. ${ }^{15}$ The time to address the LAA has come. However, while we optimize technique, the data suggest that, in most patients with AF undergoing cardiac surgery, the LAA should be eliminated.

\section{Conflict of Interest Statement}

Authors have nothing to disclose with regard to commercial support. 


\section{References}

1. Badhwar V, Rankin JS, Damiano RJ Jr, Gillinov AM, Bakaeen FG, Edgerton JR, et al. The Society of Thoracic Surgeons 2017 Clinical Practice Guidelines for the surgical treatment of atrial fibrillation. Ann Thorac Surg. 2017;103:329-41.

2. Tsai YC, Phan K, Munkholm-Larsen S, Tian DH, La Meir M, Yan TD. Surgical left atrial appendage occlusion during cardiac surgery for patients with atrial fibrillation: a meta-analysis. Eur J Cardiothorac Surg. 2015;47:847-54.

3. Elbadawi A, Olorunfemi O, Ogunbayo GO, Saad M, Elgendy IY, Arif Z, et al. Cardiovascular outcomes with surgical left atrial appendage exclusion in patients with atrial fibrillation who underwent valvular heart surgery (from the National Inpatient Sample Database). Am J Cardiol. 2017;119:2056-60.

4. Friedman DJ, Piccini JP, Want T, Zheng J, Malaisrie C, Holmes DR, et al. Association between left atrial appendage occlusion and readmission for thromboembolism among patients with atrial fibrillation undergoing concomitant cardiac surgery. JAMA. 2018;391:365-74.

5. Whitlock RRP, Healey JS, Holmes DR. Left atrial appendage occlusion debate revisited. Circulation. 2015;131:756-61.

6. Yao X, Abraham NS, Alexander GC, Crown W, Montori VM, Sangaralingham LR, et al. Effect of adherence to oral anticoagulants on risk of stroke and major bleeding among patients with atrial fibrillation. J Am Heart Assoc. 2016;5:1-12.

7. Katz ES, Tsiamtsiouris T, Applebaum RM, Schwartzbard A, Tunick PA, Kronzon I. Surgical left atrial appendage ligation is frequently incomplete: a transesophageal echocardiographic study. J Am Coll Cardiol. 2000;36:468-71.

8. García-Fernández MA, Pérez-David E, Quiles J, Peralta J, García-Rojas I, Bermejo J, et al. Role of left atrial appendage obliteration in stroke reduction in patients with mitral valve prosthesis: a transesophageal echocardiographic study. J Am Coll Cardiol. 2003;42:1253-8.

9. Healey JS, Crystal E, Lamy A, Teoh K, Semelhago L, Hohnloser SH, et al. Left Atrial Appendage Occlusion Study (LAAOS): results of a randomized controlled pilot study of left atrial appendage occlusion during coronary bypass surgery in patients at risk for stroke. Am Heart J. 2005;150:288-93.

10. Kanderian AS, Gillinov AM, Pettersson GB, Blackstone E, Klein AL. Success of surgical left atrial appendage closure: assessment by transesophageal echocardiography. J Am Coll Cardiol. 2008;52:924-9.

11. Lee R, Vassallo P, Kruse J, Malaisrie SC, Rigolin V, Andrei AC, et al. A randomized, prospective pilot comparison of 3 atrial appendage elimination techniques: internal ligation, stapled excision, and surgical excision. J Thorac Cardiovasc Surg. 2016;152:1075-80.

12. Tu HT, Campbell BC, Christensen S, Collins M, De Silva DA, Butcher KS, et al Pathophysiological determinants of worse stroke outcome in atrial fibrillation. Cerebrovasc Dis. 2010;30:389-95.

13. Lee R, Jivan A, Kruse J, McGee EC Jr, Malaisrie SC, Bernstein R, et al. Late neurologic events after surgery for atrial fibrillation: rare but relevant. Ann Thorac Surg. 2013;95:126-31.

14. Hui DS, Lee R. Modified internal ligation of the left atrial appendage. J Thorac Cardiovasc Surg. 2017:154:847-8.

15. Gammie JS, Haddad M, Milford-Beland S, Welke KF, Ferguson TB Jr, O'Brien SM, et al. Atrial fibrillation correction surgery: lessons from the Society of Thoracic Surgeons National Cardiac Database. Ann Thorac Surg. 2008;85: 909-14. 